\title{
La actividad física reduce el riesgo de diabetes tipo dos en mujeres
}

Walking compared with vigorous physical activity and risk of type 2 diabetes in women. Frank B, Hu, MD, PhD; Ronald J.Sigal, MD; Janet W, RichEdwards, ScD; Graham A.Colditz, Md, DrPH; Caren G.Solomon, MD, MPH; Walter C.Willaen, Md, DrPH; Frank E.Speizer, MD; JoAnn E Manson, MD, DrPH.

\section{Objetivo}

Examinar la relación entre la actividad física y la incidencia de diabetes tipo 2 en mujeres y comparar los beneficios de caminar vs. una actividad física vigorosa como predictores de riesgo de DBT 2.

\section{Diseño}

Estudio de cohorte prospectivo con seguimiento de 8 años consecutivos (1986-1992).

Lugar

En 11 estados de E.E.U.U. "Estudio de salud de las enfermeras".

\section{Participantes}

70.102 enfermeras mujeres entre 40 y 65 años que contestaron la encuesta en 1986 y no reportaban en ese momento enfermedad cardiovascular, cáncer o diabetes.

Una estimación de la actividad física fue obtenida a través de un cuestionario realizado en 1986 y luego actualizado en 1988 y en 1992.La confiabilidad y la validez del mismo habían sido testeadas previamente. En este cuestionario se preguntó a las mujeres, sobre la cantidad de tiempo promedio por semana que destinaban a cada una de las siguientes actividades físicas: jogging, correr, bicicleta, calistenia, aeróbica, danza aeróbica, máquina de remos, natación, jugar squash, raquetball o tenis.

Se les preguntó acerca de su paso usual al caminar, especificado como ca$\operatorname{sual}(<3.2 \mathrm{~km} / \mathrm{h})$, normal $(3.2-4.8 \mathrm{~km} / \mathrm{h})$, vivo y muy vivo $(>$ a $4.8 \mathrm{~km} / \mathrm{h})$. La correlación para el test-retest del cuestionario a 2 años fue de 0.59 .

El diagnóstico de diabetes se realizó a través de un cuestionario en donde se preguntaba acerca de síntomas, test diagnósticos y medicación, considerándose caso según criterios de la NDDG (National Diabetes Data Group).

\section{Medición de resultados}

La densidad de incidencia y el riesgo relativo de diabetes para una categoría del score de MET fue dividido por el quintilo más bajo y ajustado por edad, hipertensión, uso de reemplazo hormonal, tabaquismo, antecedentes familiares de diabetes, historia de colesterol elevado y BMI.

\section{Resultados}

Durante 8 años de seguimiento el total de casos de diabetes 2 fue de 1419 en 534.928 personas / año; con una densidad de incidencia de 265 por cada 100.000 personas / año.
Riesgo relativo con intervalo de confianza 95\% para diabetes tipo 2 según quintilos de score de actividad física. ( computado como el promedio acumulativo actualizado de 1986,1988 y 1992)

\begin{tabular}{|c|c|c|c|c|c|c|}
\hline Quintilos & 1 & 2 & 3 & 4 & 5 & \\
\hline $\begin{array}{l}\text { Mets } \\
\text { (mediana) }\end{array}$ & $\begin{array}{l}0-2.0 \\
(0.8) \\
\end{array}$ & $\begin{array}{l}2.1-4.6 \\
(3.3)\end{array}$ & $\begin{array}{l}4.7-10.4 \\
(7.7)\end{array}$ & $\begin{array}{l}10.5-21.7 \\
(15.7)\end{array}$ & $\begin{array}{l}>=21.8 \\
(35.4)\end{array}$ & $\begin{array}{l}\text { Valor de } \mathrm{P} \\
\text { por trend }\end{array}$ \\
\hline $\mathrm{N}$ de casos & 422 & 296 & 287 & 226 & 188 & \\
\hline $\begin{array}{l}\text { Personas } \\
\text { / año }\end{array}$ & 108.829 & 104.467 & 107.254 & 107.247 & 107.131 & \\
\hline $\begin{array}{l}\text { Edad } \\
\text {-ajustada }\end{array}$ & 1 & $\begin{array}{l}0.71(0.61 \\
-0.83)\end{array}$ & $\begin{array}{l}0.67(0.57 \\
-0.78)\end{array}$ & $\begin{array}{l}0.52(0.44 \\
-0.61)\end{array}$ & $\begin{array}{l}0.43(0.36 \\
-0.52)\end{array}$ & $<0.001$ \\
\hline $\begin{array}{l}\text { Multi- } \\
\text { variado }\end{array}$ & 1 & $\begin{array}{l}0.77(0.66 \\
-0.90)\end{array}$ & $\begin{array}{l}0.75(0.65 \\
-0.88)\end{array}$ & $\begin{array}{l}0.62(0.52 \\
-0.73)\end{array}$ & $\begin{array}{l}0.54(0.45 \\
-0.64) \\
\end{array}$ & $<0.001$ \\
\hline $\begin{array}{l}\text { Ajuste } \\
\text { por BMI }\end{array}$ & 1 & $\begin{array}{l}0.84(0.72 \\
-0.97)\end{array}$ & $\begin{array}{l}0.87(0.75 \\
-1.02)\end{array}$ & $\begin{array}{l}0.77(0.65 \\
-0.91)\end{array}$ & $\begin{array}{l}0.74(0.62 \\
-0.89)\end{array}$ & 0.002 \\
\hline
\end{tabular}

Se examinó luego el RR de aquellas mujeres cuya actividad física era principalmente caminar.

Riesgo relativo con intervalo de confianza $95 \%$ de diabetes 2 según quintilos de score de caminata:

\begin{tabular}{|c|c|c|c|c|c|c|}
\hline Quintilos & 1 & 2 & 3 & 4 & 5 & \\
\hline $\begin{array}{l}\text { Mets } \\
\text { (mediana) }\end{array}$ & $<=0.5(0)$ & $\begin{array}{l}0.6-2.0 \\
(1.7)\end{array}$ & $\begin{array}{l}2.1-3.8 \\
\text { (3) }\end{array}$ & $\begin{array}{l}3.9-9.9 \\
(7.5)\end{array}$ & $\begin{array}{l}>=10 \\
(20)\end{array}$ & $\begin{array}{l}\text { Valor de P } \\
\text { por trend }\end{array}$ \\
\hline $\mathrm{N}$ de casos & 250 & 205 & 145 & 133 & 111 & \\
\hline $\begin{array}{l}\text { Personas } \\
\text { / año }\end{array}$ & 62.977 & 57.069 & 52.872 & 51.277 & 51.406 & \\
\hline $\begin{array}{l}\text { Edad } \\
\text {-ajustada }\end{array}$ & 1 & $\begin{array}{l}0.88(0.73 \\
1.07)\end{array}$ & $\begin{array}{l}0.67(0.55 \\
0.83)\end{array}$ & $\begin{array}{l}0.62(0.50 \\
0.77)\end{array}$ & $\begin{array}{l}0.51(0.41 \\
0.64)\end{array}$ & $<0.001$ \\
\hline $\begin{array}{l}\text { Multi- } \\
\text { variado }\end{array}$ & 1 & $\begin{array}{l}0.91(0.75 \\
1.09)\end{array}$ & $\begin{array}{l}0.73(0.59 \\
0.90)\end{array}$ & $\begin{array}{l}0.69(0.56 \\
0.86)\end{array}$ & $\begin{array}{l}0.58(0.46 \\
0.73)\end{array}$ & $<0.001$ \\
\hline $\begin{array}{l}\text { Ajuste } \\
\text { por BMI }\end{array}$ & 1 & $\begin{array}{l}0.95(0.79 \\
1.15)\end{array}$ & $\begin{array}{l}0.80(0.65 \\
0.99)\end{array}$ & $\begin{array}{l}0.81(0.66 \\
0.01)\end{array}$ & $\begin{array}{l}0.74(0.59 \\
0.93)\end{array}$ & 0.01 \\
\hline
\end{tabular}

\section{Conclusiones}

Este estudio prospectivo sugiere que la duración e intensidad de la actividad física incluyendo el gasto de energía al caminar se asocia con menor riesgo de diabetes. La actividad física favorece el descenso y el mantenimiento del peso, también produce una disminución de la adiposidad visceral la cual se correlaciona con insulino-resistencia. En pacientes diabéticos la dieta y la caminata diaria demostró producir disminución del peso y aumento de la sensibilidad a la insulina. Cuando los gastos de energía al caminar y en la actividad física vigorosa son similares, se observaron magnitudes comparables de reducción del riesgo de diabetes.

\section{COMENTARIO}

La actividad física, incluyendo la caminata que es accesible y segura, demuestra en este estudio disminuir el riesgo de diabetes tipo 2, aún luego de ajustar el riesgo por el BMI. Por lo tanto los resultados son aplicables a mujeres con estos BMI.

Una pregunta que uno se formula a partir de estas observaciones es si pacientes obesos pero físicamente activos tienen menor morbimortalidad que aquellos con peso normal pero sedentarios. La evidencia proveniente de estudios observacionales como este demuestra que personas obe- sas pero activas tienen menor morbimortalidad que aquellas sedentarias. Este artículo apova la recomendación del CDC de realizar una actividad física de moderada intensidad de 30 minutos por día. Es importante la función del médico con respecto al consejo sobre el ejercicio. Los médicos tendemos a aconsejar esto como medida de prevención secundaria. Este consejo en jóvenes, adultos y personas libres de enfermedad representa una oportunidad para poder reforzar una medida de prevención primaria de muy bajo costo e importantísimo impacto.

Dra. Andrea Beratarrechea Becaria de gerenciamiento. Hospital Italiano de Buenos Aires.

\section{Referencias}

1-Helmrich SP, Ragland DR, Leung RW, Paffenbarger RS Jr. Physical activity and reduced occurrence of non-insulin-dependent diabetes mellitus.N Engl J Med.1991;325:147-152.

2-Manson JE, Rimm EB, Stampfer MJ, et al.Physical activity and incidence of non-insulin-dependent diabetes mellitus in women.Lancet. 1991;338:774-778.

3-Andersen RE, Wadden TA, Bartlett SJ, Zemel B, Verde TJ, Franckowiak SC. Effects of lifestyle activity vs structured aerobic exercise in obese women: a randomized tria L.JAMA.1999;281:335-340.

4-Pate RR, Pratt M, Blair SN, et al.Physical activity and public health: a recommendation from the Centers for Disease Control and Prevention and the American College of Sports Medicine.JAMA. $1995 ; 273: 402-407$

5-Blair SN, Brodney S. Effects of physical inactivity and obesity on morbidity and mortality: current evidence and research issues. Med Sci Sports Exerc. 1999 Nov;31(11 Suppl):S646-62.

6-Folsom AR, Kushi LH, Hong CP.Physical activity and incident diabetes mellitus in postmenopausal women 